\title{
Role of Laboratory in Human Brucellosis: A Case Series over 6 Years
}

\author{
Malavalli V Bhavana ${ }^{1}$, Ranjeeta Adhikary ${ }^{2}$, Hosdurg B Beena ${ }^{3}$, Aravinda Anjana ${ }^{4}$, Sangeeta Joshi ${ }^{5}$ \\ Pediatric Infectious Disease (2020): 10.5005/jp-journals-10081-1241
}

\section{Dear Editor,}

Brucellosis is a significant cause of pyrexia of unknown origin (PUO) in endemic areas. It is challenging to diagnose this infection only in clinical grounds. ${ }^{1}$ The presentation of this infection is myriad. The exposure history is often distant from the time of presentation. The incubation period is long and ranges from 2 to 5 months. ${ }^{2}$ These factors add on to the difficulty in diagnosis. The differential diagnoses are viral fever, enteric fever, connective tissue, or autoimmune disorders. We looked at the clinical and laboratory parameters in culture-confirmed human brucellosis. The purpose was to see whether any diagnostic clue can be obtained by this workup. Five patients were identified retrospectively based on positive blood cultures for Brucella species between January 2012 and December 2018. The set-up is a 600-bedded, tertiary-care hospital of South India. Blood culture was performed using BacT/ Alert 3D (Biomeriux, France). Brucella species was identified using VITEK 2 compact (Biomeriux, France). Brucella immunoglobulin M (IgM) was estimated using anti Brucella abortus IgM ELISA kit (Euroimmun, Germany). The clinical presentation, laboratory workup, and treatment protocol are elaborated in Table 1.

Brucellosis poses significant diagnostic challenges due to its nonspecific presentations. As it is clear from our data, none of our patients were suspected of brucellosis in the initial assessment. An exposure history could not be ascertained. The laboratory workup led to the final diagnosis of brucellosis. The white cell count is usually normal or slightly low as seen on complete hemogram, which suggests chronicity. Relative lymphocytosis or normal lymphocyte count is also suggested. Liver function tests usually show a mild degree of derangement in brucellosis. C-reactive protein is consistently more elevated than erythrocyte sedimentation rate, which may also help differentiate brucellosis from other chronic infections such as tuberculosis. ${ }^{3}$ Splenomegaly is consistently seen. The definitive diagnosis lies in culture. Cultures are usually positive after 48 hours of incubation. Mantur et al. have suggested that to maximize detection of the organism by the BacT/Alert, a prolonged incubation time for at least four weeks is required. ${ }^{4}$ Although the current literature says that Brucellae can be isolated within 5 days of incubation, it may still be worthwhile to keep the bottles of patients with a positive Brucella IgM for prolonged incubation. ${ }^{5}$ This may help in optimizing the recovery of this slow-growing pathogen. A prompt communication to the laboratory about suspected brucellosis patients is helpful to maintain prolonged incubation of blood culture bottles. Although IgM assays yield faster results than culture, the positive results need to be interpreted with caution. The Center of Disease Control and Prevention recommends that the serologic test results should be

\footnotetext{
${ }^{1-4}$ Department of Laboratory Medicine-Microbiology, Manipal Hospitals, Bengaluru, Karnataka, India

${ }^{5}$ Department of Microbiology, Manipal Hospitals, Dwarka, New Delhi,
} India

Corresponding Author: Malavalli V Bhavana, Department of Laboratory Medicine-Microbiology, Manipal Hospitals, Bengaluru, Karnataka, India, Phone: +91 8025023355, e-mail: bhavana224@gmail. com

How to cite this article: Bhavana MV, Adhikary R, Beena HB, et al. Role of Laboratory in Human Brucellosis: A Case Series over 6 Years. Pediatr Inf Dis 2020;2(1):34-35.

Source of support: Nil

Conflict of interest: None

confirmed using established reference testing methods and to accurately identify the presence of known risk factors. This is to be done before committing a patient to prolonged antimicrobial therapy for brucellosis or initiating public health investigations. In such a scenario, analyzing the serological test results looking into the hematological and biochemical parameters too can provide a clue about this infection. ${ }^{6}$

Common regimens for treatment include doxycycline with either rifampicin or gentamicin for 6 weeks. Trimethoprim/ sulfamethoxazole can be added as a third agent in cases of relapse. Prolonged treatment is recommended up to 6 months in neurobrucellosis or endocarditis. ${ }^{7}$

\section{References}

1. Lim ML, Rickman LS. Brucellosis. Infect Dis Clin Pract 2004;12:7-14 DOI: 10.1097/01.idc.0000104894.16995.c4.

2. Mantur BG, Amarnath SK. Brucellosis in india - a review. J Biosci 2008;33(4):539-547. DOI: 10.1007/s12038-008-0072-1.

3. Luk S, To WK. Diagnostic challenges of human brucellosis in Hong Kong: a case series in two regional hospitals. Hong Kong Med J 2010;16(4):299-303.

4. Mantur BG, Mulimani MS, Bidari LH, et al. Bacteremia is as unpredictable as clinical manifestations in human brucellosis. Int J Infect Dis 2008;12(3):303-307. DOI: 10.1016/j.ijid.2007.09.004, Epub 2007 Nov 19

5. Carrillo C, Gotuzzo E. Brucellosis. In: Schlossberg D. Clinical Infectious Disease. 2nd edn, Cambridge: Cambridge University Press; 2015. pp. 866-869.

6. https://www.cdc.gov/mmwr/preview/mmwrhtml/mm5722a3.html Accessed on 08.02.20.

7. Franco MP, Mulder M, Gilman RH, et al. Human brucellosis. Lancet Infect Dis 2007;7(12):775-786. DOI: 10.1016/S1473-3099(07)70286-4.

(0) The Author(s). 2020 Open Access This article is distributed under the terms of the Creative Commons Attribution 4.0 International License (https://creativecommons. org/licenses/by-nc/4.0/), which permits unrestricted use, distribution, and non-commercial reproduction in any medium, provided you give appropriate credit to the original author(s) and the source, provide a link to the Creative Commons license, and indicate if changes were made. The Creative Commons Public Domain Dedication waiver (http://creativecommons.org/publicdomain/zero/1.0/) applies to the data made available in this article, unless otherwise stated. 


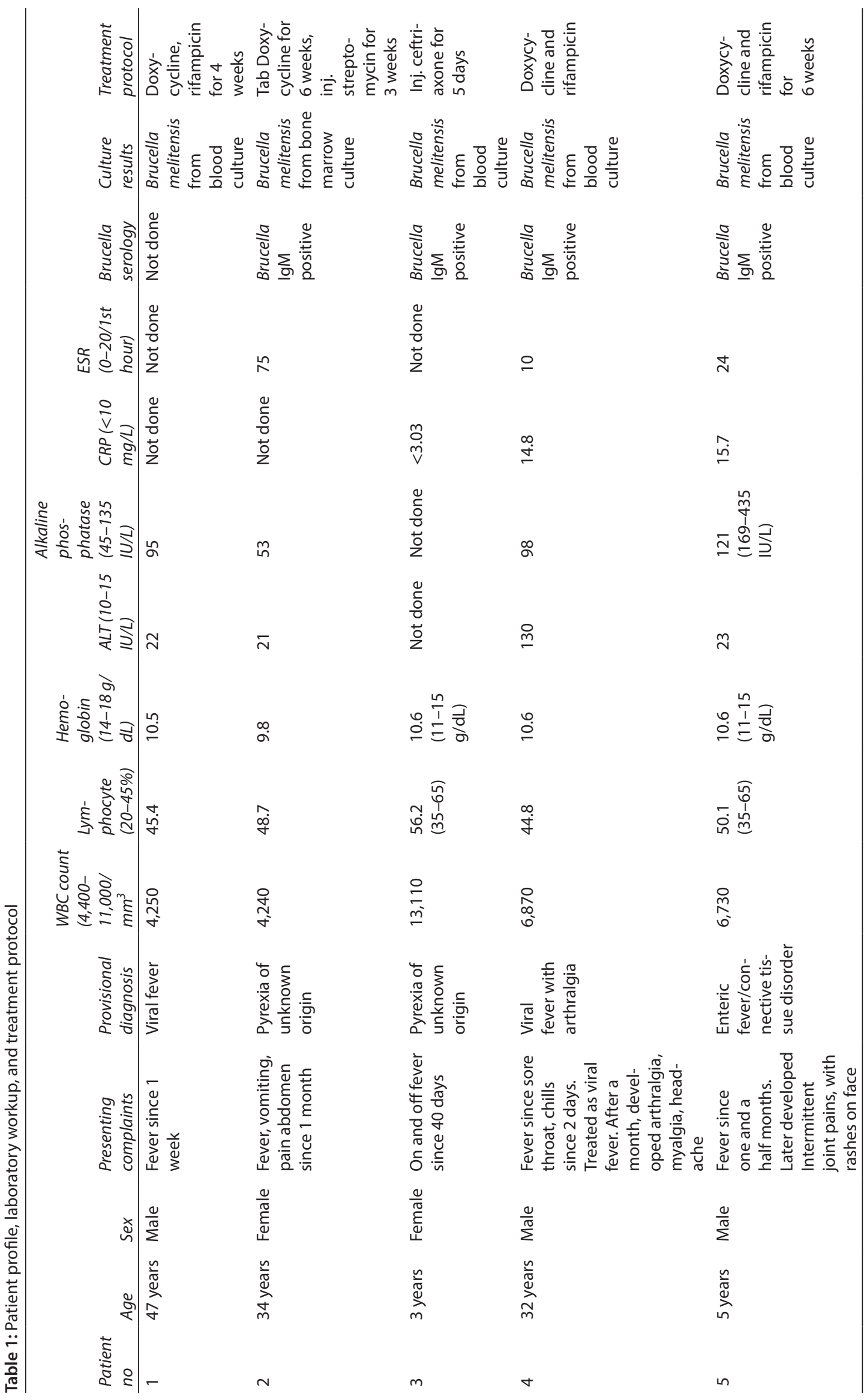

